\title{
Quantum master equation for the microcanonical ensemble
}

\author{
Massimiliano Esposito and Pierre Gaspard \\ Center for Nonlinear Phenomena and Complex Systems, Université Libre de Bruxelles, Code Postal 231, Campus Plaine, \\ B-1050 Brussels, Belgium
}

(Received 8 May 2007; published 25 October 2007)

\begin{abstract}
By using projection superoperators, we present a new derivation of the quantum master equation first obtained by the authors in Phys. Rev. E 68, 066112 (2003). We show that this equation describes the dynamics of a subsystem weakly interacting with an environment of finite heat capacity and initially described by a microcanonical distribution. After applying the rotating wave approximation to the equation, we show that the subsystem dynamics preserves the energy of the total system (subsystem plus environment) and tends towards an equilibrium state which corresponds to equipartition inside the energy shell of the total system. For infinite heat capacity environments, this equation reduces to the Redfield master equation for a subsystem interacting with a thermostat. These results should be of particular interest to describe relaxation and decoherence in nanosystems where the environment can have a finite number of degrees of freedom and the equivalence between the microcanonical and the canonical ensembles is thus not always guaranteed.
\end{abstract}

DOI: 10.1103/PhysRevE.76.041134

PACS number(s): 05.30.-d, 03.65.Yz, 76.20.+q

\section{INTRODUCTION}

Irreversible processes do not occur in isolated quantum systems with a finite number of quantum levels. In order to understand relaxation toward equilibrium in these systems, one needs to take into account the effect of their interaction with their environment. The usual way to proceed is to consider a total Hamiltonian system composed of a subsystem coupled to an environment. The subsystem dynamics is then described by the reduced density matrix of the subsystem which is obtained by tracing out the degrees of freedom of the environment from the density matrix of the total system. In order to drive the subsystem into an effective irreversible process over reasonably long-time scales (of the order of the Heisenberg time scale of the environment) on need to assume a quasi-continuous environment spectrum. This is typically valid when the energy spacing between the unperturbed quantum levels of the total system which are coupled together by the subsystem-environment interaction is sufficiently small to make the interaction between these levels effective enough to "mix" them [1]. Once this condition is satisfied, the generic way to obtain a closed master equation for the reduced density matrix of the subsystem is to use perturbation theory and the Born-Markov approximation which implicitly rely on the assumption that the environment has an infinite heat capacity and cannot be affected by the system dynamics. The environment thus plays the role of a thermostat for the subsystem and the descriptions in the canonical and microcanonical ensembles are equivalent [2]. The resulting quantum master equation is called the Redfield equation [3-7]. Since the Redfield equation can break the positivity of the subsystem density matrix it is sometimes simplified further by time averaging the equation in the interaction representation (rotating wave approximation) in order to get a master equation of Lindblad form which preserves positivity [8-12].

In the present paper, we consider situations where the environment has a quasicontinuous spectrum but a finite heat capacity. This means that the energy quanta of the subsystem may significantly affect the microcanonical temperature of the environment so that the equivalence between the microcanonical and canonical statistical ensembles is compromised and only the microcanonical ensemble can be used $a$ priori. Such situations should be generic since the density of state of a system growth exponentially with the number of degrees of freedom whereas the heat capacity only growth linearly. This means that there exists a range in the number of degrees of freedom where the quasicontinuous assumption can be satisfied without necessarily implying an infinite heat capacity. This domain where kinetic processes can occur in the subsystem but for which the usual master equations fail should be of particular importance in nanosystems where the number of degrees of freedom constituting the environment is not always large enough to be supposed infinite. In this sense, the present work can be viewed as a contribution to the recent attempts to apply statistical physics to small systems [13-18]. Our results should also be of relevance for systems which display negative heat capacities in the thermodynamic limit and to which the microcanonical ensemble description applies. A well known example is provided by coupled spin systems because their spectra remain bounded and forms bands of finite extension [19-22]. Other known examples of such systems are reported in Refs. [23-26].

The plan of the paper is the following. In Sec. II, we give a new and more enlightening derivation of the equation of Ref. [27] by using projection superoperators. In Sec. III, we apply the rotating wave approximation to our equation and prove that it conserves the energy of the total system. We also demonstrate that the subsystem relaxes to an equilibrium distribution which corresponds to equipartition inside the energy shell of the total system as expected in the microcanonical ensemble. In Sec. IV, we show that, for large heat capacity, our equation reduces to the Redfield equation and we discuss in detail the conditions under which the master equations for infinite thermostat fail to describe the correct subsystem dynamics and need to be replaced by our master equation. Conclusions are drawn in Sec. V. 


\section{NEW DERIVATION}

In this section, with the help of projection superoperators, we give a different derivation of the quantum master equation first derived in Ref. [27].

We consider the dynamics of a system with a Hamiltonian of the form

$$
H=H_{0}+\lambda V,
$$

where $\lambda$ is a small dimensionless parameter. The density matrix $\rho(t)$ of this total system obeys the von Neumann equation

$$
\begin{aligned}
\dot{\rho}(t) & =\mathcal{L} \rho(t)=-\frac{i}{\hbar}[H, \rho(t)]=\left(\mathcal{L}_{0}+\lambda \mathcal{L}^{\prime}\right) \rho(t) \\
& =-\frac{i}{\hbar}\left[H_{0}, \rho(t)\right]-\lambda \frac{i}{\hbar}[V, \rho(t)] .
\end{aligned}
$$

The solution of the von Neumann equation reads

$$
\rho(t)=\mathcal{U}(t) \rho(0)=e^{\mathcal{L} t} \rho(0) .
$$

In the interaction representation where

$$
\begin{gathered}
\rho_{I}(t)=e^{-\mathcal{L}_{0} t} \rho(t)=e^{(i / \hbar) H_{0} t} \rho(t) e^{-(i / \hbar) H_{0} t}, \\
\mathcal{L}_{I}^{\prime}(t)=e^{-\mathcal{L}_{0} t} \mathcal{L}^{\prime}(t) e^{\mathcal{L}_{0} t},
\end{gathered}
$$

the von Neumann equation takes the simple form

$$
\dot{\rho}_{I}(t)=\lambda \mathcal{L}_{I}^{\prime}(t) \rho_{I}(t)=-\lambda \frac{i}{\hbar}\left[V_{I}(t), \rho_{I}(t)\right]
$$

By integrating Eq. (6) and truncating it to order $\lambda^{2}$, we get the perturbative expansion

$$
\begin{aligned}
\rho_{I}(t) & =\mathcal{W}(t) \rho(0)=e^{-\mathcal{L}_{0} t} e^{\mathcal{L} t} \rho(0) \\
& =\left[\mathcal{W}_{0}(t)+\lambda \mathcal{W}_{1}(t)+\lambda^{2} \mathcal{W}_{2}(t)+O\left(\lambda^{3}\right)\right] \rho(0),
\end{aligned}
$$

where

$$
\begin{gathered}
\mathcal{W}_{0}(t)=I \\
\mathcal{W}_{1}(t)=\int_{0}^{t} d T \mathcal{L}_{I}^{\prime}(T) \\
\mathcal{W}_{2}(t)=\int_{0}^{t} d T \int_{0}^{T} d \tau \mathcal{L}_{I}^{\prime}(T) \mathcal{L}_{I}^{\prime}(T-\tau) .
\end{gathered}
$$

The inverse of $\mathcal{W}(t)$ reads

$$
\mathcal{W}^{-1}(t)=\mathcal{W}_{0}(t)-\lambda \mathcal{W}_{1}(t)+\lambda^{2}\left[\mathcal{W}_{1}^{2}(t)-\mathcal{W}_{2}(t)\right]+O\left(\lambda^{3}\right)
$$

Indeed, one can check that $\mathcal{W}(t) \mathcal{W}^{-1}(t)=I+O\left(\lambda^{3}\right)$. For later purpose, we also notice that

$$
\begin{aligned}
\dot{\mathcal{W}}(t) \mathcal{A} \mathcal{W}^{-1}(t)= & \lambda \dot{\mathcal{W}}_{1}(t) \mathcal{A}+\lambda^{2}\left[\dot{\mathcal{W}}_{2}(t) \mathcal{A}-\dot{\mathcal{W}}_{1}(t) \mathcal{A} \mathcal{W}_{1}(t)\right] \\
& +O\left(\lambda^{3}\right)
\end{aligned}
$$

Now, we consider a subsystem $S$ interacting with its environment $B$. The Hamiltonian of this system is given by Eq. (1) where

$$
H_{0}=H_{S}+H_{B}, \quad V=\sum_{\kappa} S^{\kappa} B_{\kappa}
$$

$S^{\kappa}\left(B_{\kappa}\right)$ is a coupling operator of system $S(B)$. We will use the index $s(b)$ to label the eigenstates of the Hamiltonian of system $S(B)$.

We will use Liouville space where operators are mapped into vectors and superoperators into matrices [28]. We recall some basic definitions

$$
\begin{gathered}
\text { scalar product: }\langle\langle A \mid B\rangle\rangle \equiv \operatorname{tr} A^{\dagger} B, \\
\text { identity: } \left.\mathcal{I} \equiv \sum_{n, n^{\prime}}\left|n n^{\prime}\right\rangle\right\rangle\left\langle\left\langle n n^{\prime}\right|,\right. \\
\left.\left|n n^{\prime}\right\rangle\right\rangle \leftrightarrow|n\rangle\left\langle n^{\prime}\right|, \quad\left\langle\left\langle n n^{\prime}|\leftrightarrow| n^{\prime}\right\rangle\langle n| .\right.
\end{gathered}
$$

Useful consequences of these definitions are

$$
\begin{gathered}
\left\langle\left\langle n n^{\prime} \mid \bar{n} \bar{n}^{\prime}\right\rangle\right\rangle=\delta_{n \bar{n}} \delta_{n^{\prime} \bar{n}^{\prime}} \\
\left\langle\left\langle n n^{\prime} \mid A\right\rangle\right\rangle=\left\langle n|A| n^{\prime}\right\rangle .
\end{gathered}
$$

An operator $A$ in the total space which can be written as a product of a system and reservoir operator $A=A_{S} \otimes A_{B}$ will read in Liouville space $\left.\left.|A\rangle\rangle=\left|A_{S}\right\rangle\right\rangle \otimes\left|A_{B}\right\rangle\right\rangle$.

We now define the following projection superoperators which act on the Liouville space of system $B$

$$
\left.\mathcal{P}=\sum_{b}|b b\rangle\right\rangle\langle\langle b b|,
$$

$$
\left.\mathcal{Q}=\sum_{b, b^{\prime}}\left|b b^{\prime}\right\rangle\right\rangle\left\langle\left\langle b b^{\prime}\right|\left(1-\delta_{b b^{\prime}}\right),\right.
$$

and which satisfy the usual properties of projection superoperators $\mathcal{P}+\mathcal{Q}=\mathcal{I}_{B}, \mathcal{P}^{2}=\mathcal{P}, \mathcal{Q}^{2}=Q$, and $\mathcal{P} \mathcal{Q}=\mathcal{Q P}=0$. $\mathcal{P}$, when acting on the density matrix of the total system, eliminates the environment coherences but keeps the environment populations and leaves unaffected the subsystem degrees of freedom. Similar projection superoperators have been recently used in Refs. [30-33]. For comparison, the projection superoperator used to derive the Redfield equation reads

$$
\left.\mathcal{P}_{\text {Red }}=\sum_{b}\left|\rho^{\mathrm{eq}}\right\rangle\right\rangle\langle\langle b b|,
$$

where $\rho^{\mathrm{eq}}$ is the equilibrium density matrix of the environment. The two projection superoperators act differently on the density matrix $\rho(t)$. In the Liouville space of the total system, we get, respectively,

$$
\begin{gathered}
\left.\mathcal{P}|\rho(t)\rangle\rangle=\sum_{b}\langle\langle b b \mid \rho(t)\rangle\rangle \otimes|b b\rangle\right\rangle, \\
\left.\left.\left.\mathcal{P}_{\text {Red }}|\rho(t)\rangle\right\rangle=\left|\rho_{S}(t)\right\rangle\right\rangle \otimes\left|\rho^{\mathrm{eq}}\right\rangle\right\rangle .
\end{gathered}
$$

$\langle\langle b b \mid \rho(t)\rangle\rangle$ is a density matrix in the system space which depends on the environment state $b$. $\mathcal{P}$ therefore correlates 
the system state with the environment state. On the contrary, $\mathcal{P}_{\text {Red }}$ assumes that the system reduced density matrix $\rho_{S}(t)$ $\equiv \Sigma_{b}\langle\langle b b \mid \rho(t)\rangle\rangle=\operatorname{tr}_{B} \rho(t)$ is independent from the environment state which always remain at equilibrium.

We now let $\mathcal{P}$ and $\mathcal{Q}$ act on the density matrix of the total system in the interaction form (7) and find

$$
\begin{gathered}
\left.\left.\mathcal{P}\left|\rho_{I}(t)\right\rangle\right\rangle=\mathcal{P W}(t)(\mathcal{P}+\mathcal{Q})\left|\rho_{I}(0)\right\rangle\right\rangle \\
\left.\left.\mathcal{Q}\left|\rho_{I}(t)\right\rangle\right\rangle=\mathcal{Q} \mathcal{W}(t)(\mathcal{P}+\mathcal{Q})\left|\rho_{I}(0)\right\rangle\right\rangle .
\end{gathered}
$$

From now on, we will consider initial conditions such that $\mathcal{Q}|\rho(0)\rangle\rangle=0$. This means that the environment part of the initial condition is diagonal in the environment eigenbasis and is thus invariant under the evolution when $\lambda=0$. Taking the time derivative of Eq. (22) and Eq. (23) and using $\left.\left.\left|\rho_{I}(0)\right\rangle\right\rangle=\mathcal{W}^{-1}(t)\left|\rho_{I}(t)\right\rangle\right\rangle$, we get

$$
\begin{aligned}
\left.\mathcal{P}\left|\dot{\rho}_{I}(t)\right\rangle\right\rangle= & \left.\mathcal{P} \dot{\mathcal{W}}(t) \mathcal{P} \mathcal{W}^{-1}(t) \mathcal{P}\left|\rho_{I}(t)\right\rangle\right\rangle \\
& \left.+\mathcal{P} \dot{\mathcal{W}}(t) \mathcal{P} \mathcal{W}^{-1}(t) \mathcal{Q}\left|\rho_{I}(t)\right\rangle\right\rangle, \\
\left.\mathcal{Q}\left|\dot{\rho}_{I}(t)\right\rangle\right\rangle= & \left.\mathcal{Q} \dot{\mathcal{W}}(t) \mathcal{P} \mathcal{W}^{-1}(t) \mathcal{P}\left|\rho_{I}(t)\right\rangle\right\rangle \\
& \left.+\mathcal{Q} \dot{\mathcal{W}}(t) \mathcal{P} \mathcal{W}^{-1}(t) \mathcal{Q}\left|\rho_{I}(t)\right\rangle\right\rangle .
\end{aligned}
$$

These equations are still exact. If we restrict ourselves to second-order perturbation theory, we can obtain the important result that the $\mathcal{P}$ projected density matrix evolution is decoupled from the $\mathcal{Q}$ projected part. Indeed, with the help of Eq. (10), we have

$$
\begin{aligned}
\mathcal{P} \dot{\mathcal{W}}(t) \mathcal{P} \mathcal{W}^{-1}(t) \mathcal{Q}= & \lambda \mathcal{P} \dot{\mathcal{W}}_{1}(t) \mathcal{P} \mathcal{Q}+\lambda^{2} \mathcal{P} \dot{\mathcal{W}}_{2}(t) \mathcal{P Q} \\
& -\lambda^{2} \mathcal{P} \dot{\mathcal{W}}_{1}(t) \mathcal{P} \mathcal{W}_{1}(t) \mathcal{Q}+O\left(\lambda^{3}\right) .
\end{aligned}
$$

The two first terms of the right-hand side are zero because $\mathcal{P Q}=0$ and the third one also because

$$
\begin{aligned}
\mathcal{P} \dot{\mathcal{W}}_{1}(t) \mathcal{P} & \left.=\sum_{b, b^{\prime}}|b b\rangle\right\rangle\left\langle\left\langle b b\left|\mathcal{L}_{I}^{\prime}(t)\right| b^{\prime} b^{\prime}\right\rangle\right\rangle\left\langle\left\langle b^{\prime} b^{\prime}\right|\right. \\
& \left.=-\frac{i}{\hbar} \sum_{\kappa} S^{\kappa}(t) \sum_{b, b^{\prime}}|b b\rangle\right\rangle\left\langle b\left|\left[B_{\kappa}(t),\left|b^{\prime}\right\rangle\left\langle b^{\prime}\right|\right]\right| b\right\rangle\left\langle\left\langle b^{\prime} b^{\prime}\right|,\right.
\end{aligned}
$$

where $\left\langle b\left|\left[B_{\kappa}(t),\left|b^{\prime}\right\rangle\left\langle b^{\prime}\right|\right]\right| b\right\rangle=0$.

After having showed that the relevant projected density matrix evolves in an autonomous way, we will now evaluate the generator of its evolution using second-order perturbation theory. Again using Eq. (10), we find that

$$
\begin{aligned}
\mathcal{P} \dot{\mathcal{W}}(t) \mathcal{P} \mathcal{W}^{-1}(t) \mathcal{P}= & \lambda \mathcal{P} \dot{\mathcal{W}}_{1}(t) \mathcal{P}+\lambda^{2} \mathcal{P} \dot{\mathcal{W}}_{2}(t) \mathcal{P} \\
& -\lambda^{2} \mathcal{P} \dot{\mathcal{W}}_{1}(t) \mathcal{P} \mathcal{W}_{1}(t) \mathcal{P}+O\left(\lambda^{3}\right) .
\end{aligned}
$$

The only term of right-hand side which is not zero is the second one [see Eq. (27)] whereupon we get

$$
\left.\left.\mathcal{P}\left|\dot{\rho}_{I}(t)\right\rangle\right\rangle=\lambda^{2} \mathcal{P} \int_{0}^{t} d \tau \mathcal{L}_{I}^{\prime}(t) \mathcal{L}_{I}^{\prime}(t-\tau) \mathcal{P}\left|\rho_{I}(t)\right\rangle\right\rangle+O\left(\lambda^{3}\right) .
$$

Now leaving the interaction representation and using the fact that $\mathcal{P} e^{-\mathcal{L}_{0} t}=e^{-\mathcal{L}_{S} t} \mathcal{P}$, we obtain

$$
\begin{aligned}
\mathcal{P}|\dot{\rho}(t)\rangle\rangle= & \left.\mathcal{L}_{S} \mathcal{P}|\rho(t)\rangle\right\rangle+\lambda^{2} e^{\mathcal{L}_{S} t} \mathcal{P} \int_{0}^{t} d \tau \mathcal{L}_{I}^{\prime}(t) \mathcal{L}_{I}^{\prime}(t-\tau) \\
& \left.\times e^{-\mathcal{L}_{S} t} \mathcal{P}|\rho(t)\rangle\right\rangle+O\left(\lambda^{3}\right) .
\end{aligned}
$$

Now, we define the quantity $P\left(E_{b}, t\right)$ that will become the fundamental quantity of our theory

$$
\frac{P\left(E_{b}, t\right)}{n\left(E_{b}\right)} \equiv\langle\langle b b|\mathcal{P}| \rho(t)\rangle\rangle,
$$

where $n\left(E_{b}\right)=\operatorname{tr}_{B} \delta\left(E_{b}-H_{B}\right)$ is the density of state of the environment. Equation (30) can thus be rewritten as

$$
\begin{aligned}
\frac{\dot{P}\left(E_{b}, t\right)}{n\left(E_{b}\right)}= & \mathcal{L}_{S} \frac{P\left(E_{b}, t\right)}{n\left(E_{b}\right)}+\lambda^{2} \sum_{b^{\prime}} \int_{0}^{t} d \tau \\
& \times e^{\mathcal{L}_{S^{t}}\left\langle\left\langle b b\left|\mathcal{L}_{I}^{\prime}(t) \mathcal{L}_{I}^{\prime}(t-\tau)\right| b^{\prime} b^{\prime}\right\rangle\right\rangle e^{-\mathcal{L}_{S^{t}} t} \frac{P\left(E_{b^{\prime}}, t\right)}{n\left(E_{b^{\prime}}\right)},}
\end{aligned}
$$

where we stop explicitly writing $+O\left(\lambda^{3}\right)$ from now on. Equation (32) can be evaluated further and we get

$$
\begin{aligned}
\frac{\dot{P}\left(E_{b}, t\right)}{n\left(E_{b}\right)}= & \mathcal{L}_{S} \frac{P\left(E_{b}, t\right)}{n\left(E_{b}\right)}-\frac{\lambda^{2}}{\hbar^{2}} \sum_{\kappa \kappa^{\prime}} \sum_{b^{\prime}} \int_{0}^{t} d \tau e^{-(i / \hbar) H_{S^{t}}} \\
& \times\langle b|\left[S^{\kappa}(t) B_{\kappa}(t),\left[S^{\kappa^{\prime}}(t-\tau) B_{\kappa^{\prime}}(t-\tau),\right.\right. \\
& e^{\left.\left.(i / \hbar) H_{S^{t}} \frac{P\left(E_{b^{\prime}}, t\right)}{n\left(E_{b^{\prime}}\right)} e^{-(i / \hbar) H_{S^{t}} \mid}\left|b^{\prime}\right\rangle\left\langle b^{\prime}\right|\right]\right]|b\rangle e^{(i / \hbar) H_{S^{t}}} .}
\end{aligned}
$$

By evaluating the commutators, we get the non-Markovian version of our master equation for environments with discrete spectra

$$
\begin{aligned}
& \frac{\dot{P}\left(E_{b}, t\right)}{n\left(E_{b}\right)}=\mathcal{L}_{S} \frac{P\left(E_{b}, t\right)}{n\left(E_{b}\right)}-\frac{\lambda^{2}}{\hbar^{2}} \sum \sum_{\kappa \kappa^{\prime}} \int_{b^{\prime}}^{t} d \tau \\
& \times\left\{+e^{i \omega_{b b^{\prime}} \tau}\left\langle b\left|B_{\kappa}\right| b^{\prime}\right\rangle\left\langle b^{\prime}\left|B_{\kappa^{\prime}}\right| b\right\rangle S^{\kappa} S^{\kappa^{\prime}}(-\tau) \frac{P\left(E_{b}, t\right)}{n\left(E_{b}\right)}\right. \\
& -e^{i \omega_{b b^{\prime}} \tau}\left\langle b\left|B_{\kappa}\right| b^{\prime}\right\rangle\left\langle b^{\prime}\left|B_{\kappa^{\prime}}\right| b\right\rangle S^{\kappa} \frac{P\left(E_{b^{\prime}}, t\right)}{n\left(E_{b^{\prime}}\right)} S^{\kappa^{\prime}}(-\tau) \\
& -e^{-i \omega_{b b^{\prime}} \tau}\left\langle b\left|B_{\kappa^{\prime}}\right| b^{\prime}\right\rangle\left\langle b^{\prime}\left|B_{\kappa}\right| b\right\rangle S^{\kappa^{\prime}}(-\tau) \frac{P\left(E_{b^{\prime}}, t\right)}{n\left(E_{b^{\prime}}\right)} S^{\kappa}
\end{aligned}
$$




$$
\left.+e^{-i \omega_{b b^{\prime}} \tau}\left\langle b\left|B_{\kappa^{\prime}}\right| b^{\prime}\right\rangle\left\langle b^{\prime}\left|B_{\kappa}\right| b\right\rangle \frac{P\left(E_{b}, t\right)}{n\left(E_{b}\right)} S^{\kappa^{\prime}}(-\tau) S^{\kappa}\right\},
$$

where $\omega_{b b^{\prime}}=\left(E_{b}-E_{b^{\prime}}\right) / \hbar$ are the Bohr frequencies of the environment.

We assume now that the spectra of the environment is dense enough to be treated as quasicontinuum so that we can use the following equivalences

$$
\begin{gathered}
P\left(E_{b}, t\right)=n\left(E_{b}\right) \operatorname{tr}_{B}|b\rangle\langle b| \rho(t) \rightarrow P(\epsilon, t)=\operatorname{tr}_{B} \delta\left(\epsilon-H_{B}\right) \rho(t), \\
\sum_{b^{\prime}} \rightarrow \int d \epsilon^{\prime} n\left(\epsilon^{\prime}\right), \quad n\left(E_{b}\right) \rightarrow n(\epsilon), \quad\left\langle b|B| b^{\prime}\right\rangle \rightarrow\left\langle\epsilon|B| \epsilon^{\prime}\right\rangle .
\end{gathered}
$$

The non-Markovian version of our master equation for environments with a quasicontinuous spectrum is therefore

$$
\begin{aligned}
\dot{P}(\epsilon, t)= & \mathcal{L}_{S} P(\epsilon, t)-\frac{\lambda^{2}}{\hbar^{2}} \sum_{\kappa \kappa^{\prime}} \int d \epsilon^{\prime} \int_{0}^{t} d \tau \\
& \times\left\{+e^{i\left(\epsilon-\epsilon^{\prime}\right) \tau / \hbar} n\left(\epsilon^{\prime}\right)\left\langle\epsilon\left|B_{\kappa}\right| \epsilon^{\prime}\right\rangle\left\langle\epsilon^{\prime}\left|B_{\kappa^{\prime}}\right| \epsilon\right\rangle S^{\kappa}\right. \\
& \times S^{\kappa^{\prime}}(-\tau) P(\epsilon, t) \\
& -e^{i\left(\epsilon-\epsilon^{\prime}\right) \tau / \hbar} n(\epsilon)\left\langle\epsilon\left|B_{\kappa}\right| \epsilon^{\prime}\right\rangle\left\langle\epsilon^{\prime}\left|B_{\kappa^{\prime}}\right| \epsilon\right\rangle S^{\kappa} P\left(\epsilon^{\prime}, t\right) S^{\kappa^{\prime}}(-\tau) \\
& -e^{-i\left(\epsilon-\epsilon^{\prime}\right) \tau / \hbar} n(\epsilon)\left\langle\epsilon\left|B_{\kappa^{\prime}}\right| \epsilon^{\prime}\right\rangle\left\langle\epsilon^{\prime}\left|B_{\kappa}\right| \epsilon\right\rangle S^{\kappa^{\prime}}(-\tau) P\left(\epsilon^{\prime}, t\right) S^{\kappa} \\
& +e^{-i\left(\epsilon-\epsilon^{\prime}\right) \tau / \hbar} n\left(\epsilon^{\prime}\right)\left\langle\epsilon\left|B_{\kappa^{\prime}}\right| \epsilon^{\prime}\right\rangle\left\langle\epsilon^{\prime}\left|B_{\kappa}\right| \epsilon\right\rangle P(\epsilon, t) \\
& \left.\times S^{\kappa^{\prime}}(-\tau) S^{\kappa}\right\} .
\end{aligned}
$$

This equation was first obtained in Ref. [27]. Notice that the reduced density matrix of the subsystem is obtained from the quantity $P(\epsilon, t)$ using

$$
\rho_{S}(t)=\operatorname{tr}_{B} \rho(t)=\int d \epsilon P(\epsilon, t) .
$$

The quantity $P(\epsilon, t)$ can be seen as an environment energy distributed subsystem density matrix. One should also realize that Eq. (36) is a closed equation for the $P(\epsilon, t)$ 's but cannot be converted without approximations into a closed equation for $\rho_{S}(t)$ using Eq. (37). This is a first indication that Eq. (36) contains more information than what can be contained in a closed equation for $\rho_{S}(t)$.

In the environment space, the equilibrium correlation function between two coupling operators $B_{\kappa}$ and $B_{\kappa^{\prime}}$ is

$$
\alpha_{\kappa \kappa^{\prime}}(t)=\operatorname{tr}_{B} \rho^{\mathrm{eq}} e^{(i / \hbar) H_{B} t} B_{\kappa} e^{-(i / \hbar) H_{B} t} B_{\kappa^{\prime}},
$$

where $\rho^{\mathrm{eq}}$ is the equilibrium density matrix of the environment. If the environment is described by a microcanonical distribution at energy $\epsilon$

$$
\rho_{\text {mic }}^{\mathrm{eq}}(\epsilon)=\delta\left(\epsilon-H_{B}\right) / n(\epsilon),
$$

the correlation function reads

$$
\alpha_{\kappa \kappa^{\prime}}(\epsilon, t)=\int d \epsilon^{\prime} n\left(\epsilon^{\prime}\right) e^{i\left(\epsilon-\epsilon^{\prime}\right) t / \hbar}\left\langle\epsilon\left|B_{\kappa}\right| \epsilon^{\prime}\right\rangle\left\langle\epsilon^{\prime}\left|B_{\kappa^{\prime}}\right| \epsilon\right\rangle .
$$

Its Fourier transform $\left[\tilde{\alpha}(\omega)=\int_{-\infty}^{\infty}(d t / 2 \pi) e^{i \omega t} \alpha(t)\right]$ is

$$
\tilde{\alpha}_{\kappa \kappa^{\prime}}(\epsilon, \omega)=\hbar n(\epsilon+\hbar \omega)\left\langle\epsilon\left|B_{\kappa}\right| \epsilon+\hbar \omega\right\rangle\left\langle\epsilon+\hbar \omega\left|B_{\kappa^{\prime}}\right| \epsilon\right\rangle .
$$

This quantity has a useful physical interpretation. $\Sigma_{\kappa \kappa^{\prime}} \widetilde{\alpha}_{\kappa \kappa^{\prime}}$ is, up to a factor $\hbar^{2} /\left(\pi \lambda^{2}\right)$, the Fermi golden rule transition rate for the environment in a microcanonical distribution at energy $\epsilon$ to absorb (emit) a quantum of energy $\hbar \omega$ when submitted to a periodic perturbation $\Sigma_{\kappa} B_{\kappa} \cos \omega t$. Using Eq. (41), we can easily verify the important property

$$
\widetilde{\alpha}_{\kappa \kappa^{\prime}}(\epsilon, \omega) n(\epsilon)=\widetilde{\alpha}_{\kappa^{\prime} \kappa}(\epsilon+\hbar \omega,-\omega) n(\epsilon+\hbar \omega) .
$$

We can now rewrite Eq. (36) as

$$
\begin{aligned}
\dot{P}(\epsilon, t)= & \mathcal{L}_{S} P(\epsilon, t)+\frac{\lambda^{2}}{\hbar^{2}} \sum_{\kappa \kappa^{\prime}} \int_{0}^{t} d \tau \int_{-\infty}^{\infty} d \omega \\
& \times\left\{-e^{-i \omega \tau} \widetilde{\alpha}_{\kappa \kappa^{\prime}}(\epsilon, \omega) S^{\kappa} S^{\kappa^{\prime}}(-\tau) P(\epsilon, t)\right. \\
& -e^{i \omega \tau} \widetilde{\alpha}_{\kappa^{\prime} \kappa}(\epsilon, \omega) P(\epsilon, t) S^{\kappa^{\prime}}(-\tau) S^{\kappa} \\
& +e^{-i \omega \tau} \widetilde{\alpha}_{\kappa^{\prime} \kappa}(\epsilon+\hbar \omega,-\omega) S^{\kappa} P(\epsilon+\hbar \omega, t) S^{\kappa^{\prime}}(-\tau) \\
& \left.+e^{i \omega \tau} \widetilde{\alpha}_{\kappa \kappa^{\prime}}(\epsilon+\hbar \omega,-\omega) S^{\kappa^{\prime}}(-\tau) P(\epsilon+\hbar \omega, t) S^{\kappa}\right\} .
\end{aligned}
$$

This equation is the same non-Markovian master equation as Eq. (36) but written in a more compact and intuitive form. It is explicit now that the effect of the environment on the subsystem dynamics only enters the description via the environment microcanonical correlation function. In standard master equations, the same is true but with canonical instead of microcanonical correlation functions. The Markovian version of this equation is obtained by taking the upper bound of the integral to infinity $\int_{0}^{t} \rightarrow \int_{0}^{\infty}$. This approximation is well known in the literature and is justified when the environment correlation function decays on time scales $\tau_{c}$ much shorter then the fastest time scale of the free subsystem evolution $\tau_{S}$ (typically given by the inverse of the largest subsystem Bohr frequency).

A remark concerning the terminology is required at this point. In our terminology, the generator of time evolution is said non-Markovian if it explicitly depends on time whether or not it acts on $\rho_{S}$ or $P(\epsilon)$. An alternative definition (see [33]) defines the system dynamics as non-Markovian if it cannot be described by a time-independent generator acting on $\rho_{S}$. With this definition, Eq. (43) with the upper bound of the time integral taken to infinity would describe a nonMarkovian dynamics.

\section{ROTATING WAVE APPROXIMATION}

In this section, we will use the rotating wave approximation (RWA) which consist in time averaging the Markovian 
version of the master equation in the interaction form. When applying this approximation to Eq. (43), the equation takes a simple form which allows to prove important results.

The RWA is most commonly used in quantum optic $[7,11,12]$ because the free subsystem dynamics generally evolves on times scales $\tau_{S}$ which are much faster than the relaxation time scales $\tau_{r}$ induced by the coupling to the environment. The master equation in the interacting representation evolves then very slowly compared to the Bohr frequencies of the subsystem which can therefore be averaged out. In other words, the RWA is justified if the time scale separation $\tau_{c} \ll \tau_{S} \ll \tau_{r}$ exist. In the mathematical physics, peoples usually refer to this averaging procedure (which is performed after a rescaling of time $t^{\prime}=\lambda^{2} t$ ) as the weak cou- pling limit $[9,10]$ since the smaller the coupling the longer $\tau_{r}$. Their motivation is to impose a Lindblad form to the master equation generator in order to ensure the positivity of the subsystem density matrix $[7,10,29]$. The same situation occurs in our case. In Ref. [33], Breuer has generalized the Lindblad theory to generators which act on projected total density matrices of the type (20) which correlate the systemreservoir dynamics. Equation (43) is not of the generalized Lindblad form and could in principle lead to positivity breakdown similarly as the Redfield equation [6,34-36]. The RWA can be used to guaranty that our equation preserves the positivity of the subsystem density matrix. By writing Eq. (43) in the interaction representation and by projecting the resulting equation in the subsystem eigenbasis, we get

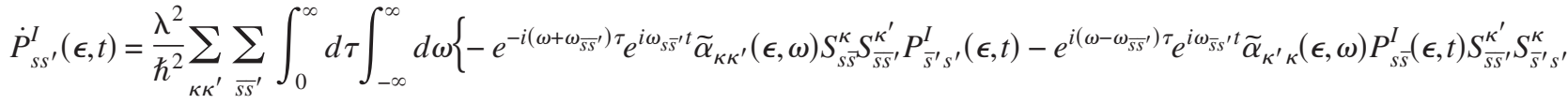

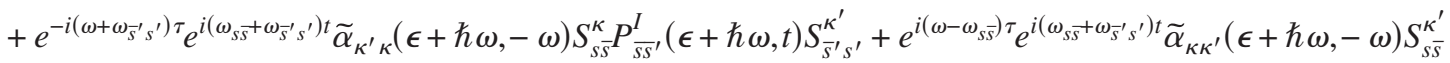

$$
\begin{aligned}
& \left.\times P_{\overline{s s^{\prime}}}^{I}(\epsilon+\hbar \omega, t) S_{\bar{s}^{\prime} s^{\prime}}^{\kappa}\right\} .
\end{aligned}
$$

The RWA consist in time averaging $\lim _{T \rightarrow \infty}(1 / 2 T) \int_{-T}^{T} d t$ the right-hand side of Eq. (44) so that

$$
\begin{aligned}
& \dot{P}_{s s^{\prime}}^{I}(\epsilon, t)=\frac{\lambda^{2}}{\hbar^{2}} \sum_{\kappa \kappa^{\prime}} \sum_{\overline{s s^{\prime}}} \int_{0}^{\infty} d \tau \int_{-\infty}^{\infty} d \omega\left\{-\delta_{\bar{s} \bar{s}^{\prime}} e^{-i\left(\omega+\omega_{\bar{s} s}\right) \tau} \widetilde{\alpha}_{\kappa \kappa^{\prime}}(\epsilon, \omega) S_{s \bar{s}}^{\kappa} S_{\bar{s} s}^{\kappa^{\prime}} P_{s s^{\prime}}^{I}(\epsilon, t)-\delta_{\bar{s} s^{\prime}} e^{i\left(\omega-\omega_{s^{\prime} \bar{s}^{\prime}}\right) \tau} \widetilde{\alpha}_{\kappa^{\prime} \kappa}(\epsilon, \omega) P_{s s^{\prime}}^{I}(\epsilon, t) S_{s^{\prime} \bar{s}^{\prime}}^{\kappa^{\prime}} S_{\bar{s}^{\prime} s^{\prime}}^{\kappa}\right. \\
& +\left[\left(1-\delta_{s s^{\prime}}\right) \delta_{s \bar{s}} \delta_{\bar{s}^{\prime} s^{\prime}}+\delta_{s s^{\prime}} \delta_{\bar{s}^{\prime} \bar{s}}\right] e^{-i\left(\omega+\omega_{\bar{s}^{\prime} s^{\prime}}\right) \tau} \widetilde{\alpha}_{\kappa^{\prime} \kappa}(\epsilon+\hbar \omega,-\omega) S_{s \bar{s}}^{\kappa} P_{\overline{s s^{\prime}}}^{I}(\epsilon+\hbar \omega, t) S_{\overline{s^{\prime}} s^{\prime}}^{\kappa^{\prime}} \\
& \left.+\left[\left(1-\delta_{s s^{\prime}}\right) \delta_{s \bar{s}} \delta_{\bar{s}^{\prime} s^{\prime}}+\delta_{s s^{\prime}} \delta_{\bar{s}^{\prime}}\right] e^{i\left(\omega-\omega_{s \bar{s}}\right) \tau} \widetilde{\alpha}_{\kappa \kappa^{\prime}}(\epsilon+\hbar \omega,-\omega) S_{s \bar{s}}^{\kappa^{\prime}} P_{\overline{s s^{\prime}}}^{I}(\epsilon+\hbar \omega, t) S_{\bar{s}^{\prime} s^{\prime}}^{\kappa}\right\} .
\end{aligned}
$$

Using $\int_{0}^{\infty} d \tau e^{ \pm i \omega \tau}=\pi \delta(\omega) \pm i P(1 / \omega)$, we finally get the Markovian version of our master equation in the RWA

$$
\begin{aligned}
& \dot{P}_{s s^{\prime}}^{I}(\epsilon, t)=\frac{\lambda^{2}}{\hbar^{2}} \sum_{\kappa \kappa^{\prime}} \sum_{\overline{s s^{\prime}}}\left\{-\pi \delta_{s \bar{s}^{\prime}} \widetilde{\alpha}_{\kappa \kappa^{\prime}}\left(\epsilon,-\omega_{\overline{s s}}\right) S_{s \bar{s}}^{\kappa} S_{\overline{s s}}^{\kappa^{\prime}} P_{s s^{\prime}}^{I}(\epsilon, t)-\pi \delta_{\bar{s} s^{\prime}} \widetilde{\alpha}_{\kappa^{\prime} \kappa}\left(\epsilon, \omega_{s^{\prime} \bar{s}^{\prime}}\right) P_{s s^{\prime}}^{I}(\epsilon, t) S_{s^{\prime} \bar{s}^{\prime}}^{\kappa^{\prime}} S_{\bar{s}^{\prime} s^{\prime}}^{\kappa}\right. \\
& +\pi\left[\left(1-\delta_{s s^{\prime}}\right) \delta_{s \bar{s}} \delta_{\bar{s}^{\prime} s^{\prime}}+\delta_{s s^{\prime}} \delta_{\bar{s}^{\prime} \bar{s}}\right] \widetilde{\alpha}_{\kappa^{\prime} \kappa}\left(\epsilon-\hbar \omega_{\overline{s^{\prime}} s^{\prime}}, \omega_{\bar{s}^{\prime} s^{\prime}}\right) S_{s \bar{s}}^{\kappa} P_{\overline{s s^{\prime}}}^{I}\left(\epsilon-\hbar \omega_{\bar{s}^{\prime} s^{\prime}}, t\right) S_{\bar{s}^{\prime} s^{\prime}}^{\kappa^{\prime}}
\end{aligned}
$$

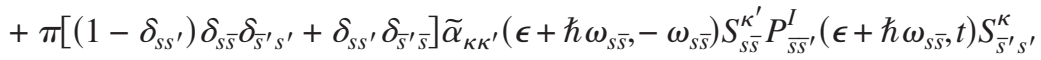

$$
\begin{aligned}
& +i \delta_{s \bar{s}^{\prime}} \int_{-\infty}^{\infty} d \omega \mathrm{P} \frac{\widetilde{\alpha}_{\kappa \kappa^{\prime}}(\epsilon, \omega)}{\omega+\omega_{\bar{s} s}} S_{s \bar{s}}^{\kappa} S_{\bar{s} s}^{\kappa^{\prime}} P_{s s^{\prime}}^{I}(\epsilon, t)-i \delta_{\bar{s} s^{\prime}} \int_{-\infty}^{\infty} d \omega \mathrm{P} \frac{\widetilde{\alpha}_{\kappa^{\prime} \kappa}(\epsilon, \omega)}{\omega-\omega_{s^{\prime} \bar{s}^{\prime}}} P_{s s^{\prime}}^{I}(\epsilon, t) S_{s^{\prime} \bar{s}^{\prime}}^{\kappa^{\prime}} S_{\bar{s}^{\prime} s^{\prime}}^{\kappa} \\
& -i\left[\left(1-\delta_{s s^{\prime}}\right) \delta_{s \bar{s}} \delta_{\bar{s}^{\prime} s^{\prime}}+\delta_{s s^{\prime}} \delta_{\bar{s}^{\prime} \bar{s}}\right] S_{s \bar{s}}^{\kappa} \int_{-\infty}^{\infty} d \omega \mathrm{P} \frac{\widetilde{\alpha}_{\kappa^{\prime} \kappa}(\epsilon+\hbar \omega,-\omega) P_{\overline{s s^{\prime}}}^{I}(\epsilon+\hbar \omega, t)}{\omega+\omega_{\bar{s} s^{\prime}}} S_{\bar{s}^{\prime} s^{\prime}}^{\kappa^{\prime}} \\
& \left.+i\left[\left(1-\delta_{s s^{\prime}}\right) \delta_{s \bar{s}} \delta_{\bar{s}^{\prime} s^{\prime}}+\delta_{s s^{\prime}} \delta_{\bar{s}^{\prime} \bar{s}}\right] S_{s \bar{s}}^{\kappa^{\prime}} \int_{-\infty}^{\infty} d \omega \mathrm{P} \frac{\widetilde{\alpha}_{\kappa \kappa^{\prime}}(\epsilon+\hbar \omega,-\omega) P_{\overline{s s^{\prime}}}^{I}(\epsilon+\hbar \omega, t)}{\omega-\omega_{s \bar{s}}} S_{\overline{\bar{s}^{\prime}} s^{\prime}}^{\kappa}\right\} .
\end{aligned}
$$

This equation is a central result of this paper. It might look a little complicated but is in fact relatively simple. The first four terms are responsible for the damping of the subsystem and the four last term are small shifts in the Bohr frequencies of the subsystem. The populations $\left(s=s^{\prime}\right)$ evolve independently from the coherences $\left(s \neq s^{\prime}\right)$ and the coherences evolve independently for each other following exponentially damped oscillations

$$
\dot{P}_{s s^{\prime}}(\epsilon, t)=\left(-\Gamma_{s s^{\prime}}-i \Omega_{s s^{\prime}}\right) P_{s s^{\prime}}(\epsilon, t),
$$

where the damping rates are given by 


$$
\Gamma_{s s^{\prime}}=\frac{\lambda^{2}}{\hbar^{2}} \sum_{\kappa \kappa^{\prime}}\left\{-2 \pi \widetilde{\alpha}_{\kappa \kappa^{\prime}}(\epsilon, 0) S_{s s}^{\kappa^{\prime}} S_{s^{\prime} s^{\prime}}^{\kappa}+\pi \sum_{\bar{s}}\left[\widetilde{\alpha}_{\kappa \kappa^{\prime}}\left(\epsilon, \omega_{s \bar{s}}\right) S_{s \bar{s}}^{\kappa} S_{\bar{s} s}^{\kappa^{\prime}}+\widetilde{\alpha}_{\kappa^{\prime} \kappa}\left(\epsilon, \omega_{s^{\prime} \bar{s}}\right) S_{s^{\prime} \bar{s}}^{\kappa^{\prime}} S_{\bar{s} s^{\prime}}^{\kappa}\right]\right\}
$$

and where the modified Bohr frequencies are given by

$$
\begin{aligned}
\Omega_{s s^{\prime}}= & \omega_{s s^{\prime}}-\frac{\lambda^{2}}{\hbar^{2}} \sum_{\kappa \kappa^{\prime}} \sum_{\bar{s}}\left[\int_{-\infty}^{\infty} d \omega \mathrm{P} \frac{\widetilde{\alpha}_{\kappa \kappa^{\prime}}(\epsilon, \omega)}{\omega+\omega_{\bar{s} s}} S_{s \bar{s}}^{\kappa} S_{\bar{s} s}^{\kappa^{\prime}}\right. \\
& \left.-\int_{-\infty}^{\infty} d \omega \mathrm{P} \frac{\widetilde{\alpha}_{\kappa \kappa^{\prime}}(\epsilon, \omega)}{\omega+\omega_{\bar{s} s^{\prime}}} S_{s^{\prime} \bar{s}}^{\kappa} S_{\bar{s} s^{\prime}}^{\kappa^{\prime}}\right] .
\end{aligned}
$$

This equation is local in the energy of the environment. This is not the case of the equation which rules the population dynamics

$$
\begin{aligned}
\dot{P}_{s s}(\epsilon, t)= & \frac{\lambda^{2}}{\hbar^{2}} \sum_{\kappa \kappa^{\prime}} \sum_{\bar{s}}\left\{-2 \pi \widetilde{\alpha}_{\kappa \kappa^{\prime}}\left(\epsilon,-\omega_{\bar{s} s}\right) S_{s \bar{s}}^{\kappa} S_{\bar{s} s}^{\kappa^{\prime}} P_{s s}(\epsilon, t)\right. \\
& \left.+2 \pi \widetilde{\alpha}_{\kappa^{\prime} \kappa}\left(\epsilon-\hbar \omega_{\overline{s s}}, \omega_{\bar{s} s}\right) S_{s \bar{s}}^{\kappa} S_{\bar{s} s}^{\kappa^{\prime}} P_{\overline{s s}}\left(\epsilon-\hbar \omega_{\overline{s s}}, t\right)\right\} .
\end{aligned}
$$

This equation is a kind of Pauli equation for the total system which preserves the unperturbed energy of the total system

$$
\left\langle H_{0}\right\rangle_{t}=\int d \epsilon \sum_{s}\left(E_{s}+\epsilon\right) P_{s s}(\epsilon, t)=\int d \varepsilon \varepsilon f(\varepsilon)
$$

where

$$
f(\varepsilon)=\sum_{s} P_{s s}\left(\varepsilon-E_{s}, t\right)
$$

represents the probability distribution inside a given energy shell of energy $\varepsilon$ of the unperturbed total system. Indeed, using Eq. (50), we find

$$
\dot{f}(\varepsilon)=\sum_{s} \dot{P}_{s s}\left(\varepsilon-E_{s}, t\right)=0 .
$$

This shows that the energy of the total system is conserved by the dynamics because the probability is preserved inside each energy shell of the total system. If the initial condition of the total system is a product of a subsystem pure state of energy $E_{s}$ with a microcanonical distribution at energy $\epsilon_{0}$ for the environment $\left[P(\epsilon, 0)=|s\rangle\langle s| \delta\left(\epsilon-\epsilon_{0}\right)\right]$ the energy distribution of the total system corresponds to $f(\varepsilon)=\delta\left(\varepsilon-E_{s}-\epsilon_{0}\right)$. If the subsystem is initially described by a density matrix $\rho_{S}(0)$, we get $f(\varepsilon)=\Sigma_{s} \rho_{S, s s}(0) \delta\left(\varepsilon-E_{s}-\epsilon_{0}\right)$. The population dynamics independently evolves in each energy shell of the total system. The equilibrium distribution of Eq. (50) is

$$
\frac{P_{\overline{s s}}\left(\epsilon-\hbar \omega_{\overline{s s}}, \infty\right)}{P_{s s}(\epsilon, \infty)}=\frac{\widetilde{\alpha}_{\kappa \kappa^{\prime}}\left(\epsilon,-\omega_{\overline{s s}}\right)}{\widetilde{\alpha}_{\kappa^{\prime} \kappa}\left(\epsilon-\hbar \omega_{\bar{s} s}, \omega_{\overline{s s}}\right)} .
$$

Using Eq. (42) and $\varepsilon=\epsilon+E_{s}$, Eq. (54) becomes

$$
\frac{P_{\overline{s s}}\left(\varepsilon-E_{\bar{s}}, \infty\right)}{P_{s s}\left(\varepsilon-E_{s}, \infty\right)}=\frac{n\left(\varepsilon-E_{\bar{s}}\right)}{n\left(\varepsilon-E_{s}\right)},
$$

which expresses detailed balance at equilibrium. Because $f(\varepsilon)$ is invariant under the dynamics, we finally get

$$
P_{s s}\left(\varepsilon-E_{s}, \infty\right)=\frac{n\left(\varepsilon-E_{s}\right)}{\sum_{\bar{s}} n\left(\varepsilon-E_{\bar{S}}\right)} f(\varepsilon) .
$$

At equilibrium, each quantum level inside a given energy shell of the total system has the same probability. This means that our equation describes how any initial distribution inside a given energy shell of the total system reaches equilibrium.

\section{EQUIVALENCE BETWEEN THE ENSEMBLES}

In this section, we start by showing using a simple qualitative argument that if the environment is initially described by a canonical distribution and is assumed large enough for not being affected by the subsystem dynamics, our equation reduces to the Redfield master equation. However, the most important part of this section is devoted to the problem of understanding in detail how our master equation (which rules the dynamics of a subsystem interacting with an environment described by a microcanonical distribution) effectively reduces to a Redfield master equation (which rules the dynamics of a subsystem interacting with an environment described by a canonical distribution) if the equivalence between the microcanonical and the canonical ensemble is satisfied for the environment.

The canonical density matrix of the environment is related to the microcanonical density matrix of the environment by

$$
\rho_{\mathrm{can}}^{\mathrm{eq}}(\beta)=\frac{e^{-\beta H_{B}}}{Z}=\int d \epsilon W(\epsilon) \rho_{\mathrm{mic}}^{\mathrm{eq}}(\epsilon)
$$

where

$$
W(\epsilon)=\frac{e^{-\beta \epsilon} n(\epsilon)}{Z} .
$$

We notice that the condition (42) found for the microcanonical correlation functions is in fact at the origin of the KMS condition for the canonical correlation functions. Indeed, using Eq. (42) and

$$
\widetilde{\alpha}_{\kappa \kappa^{\prime}}(\beta, \omega)=\int d \epsilon W(\epsilon) \widetilde{\alpha}_{\kappa \kappa^{\prime}}(\epsilon, \omega),
$$

we obtain the KMS condition

$$
\widetilde{\alpha}_{\kappa \kappa^{\prime}}(\beta, \omega)=e^{\beta \hbar \omega} \widetilde{\alpha}_{\kappa^{\prime} \kappa}(\beta,-\omega) .
$$

A very easy way to see the link between our equation and the Redfield equation is to assume that the environment is ini- 
tially described by a canonical distribution and remains in it at any time during its interaction with the subsystem. This assumption can be expressed by the ansatz

$$
P(\epsilon, t) \approx \rho_{S}(t) \frac{e^{-\beta \epsilon} n(\epsilon)}{Z} .
$$

This can be qualitatively justified by assuming that the environment is very large compared to the subsystem. By integrating Eq. (43) over the energy of the environment and with the help of Eq. (37), Eq. (59), and Eq. (61), we get the Redfield equation [3-7]

$$
\begin{aligned}
\dot{\rho}_{S}(t)= & \mathcal{L}_{S} \rho_{S}(t)+\frac{\lambda^{2}}{\hbar^{2}} \sum_{\kappa \kappa^{\prime}} \int_{0}^{t} d \tau\left\{-\alpha_{\kappa \kappa^{\prime}}(\beta, \tau) S^{\kappa} S^{\kappa^{\prime}}(-\tau) \rho_{S}(t)\right. \\
& -\alpha_{\kappa \kappa^{\prime}}^{*}(\beta, \tau) \rho_{S}(t) S^{\kappa^{\prime}}(-\tau) S^{\kappa} \\
& +\alpha_{\kappa \kappa^{\prime}}^{*}(\beta, \tau) S^{\kappa} \rho_{S}(t) S^{\kappa^{\prime}}(-\tau) \\
& \left.+\alpha_{\kappa \kappa^{\prime}}(\beta, \tau) S^{\kappa^{\prime}}(-\tau) \rho_{S}(t) S^{\kappa}\right\}
\end{aligned}
$$

with $\alpha(\tau)=\int d \omega e^{-i \omega \tau} \alpha(\omega)$. Exactly the same procedure can be used for Eq. (46) and we thus get the RWA version of the Redfield equation (also called the weak-coupling-limit master equation) [7,9-12]. In this last case, one can also show that, by integrating the equilibrium distribution (54) over the energy of the environment and by using (61) and the KMS condition (60), we get

$$
\frac{\rho_{\overline{s s}}^{S}}{\rho_{s s}^{S}}=e^{-\beta \hbar \omega_{\overline{s s}}} .
$$

As expected, using the normalization condition $\Sigma_{\bar{s}} \rho_{\overline{s s}}=1$, we find that the subsystem equilibrium distribution of the RWA form of the Redfield equation is a canonical distribution at the temperature of the environment

$$
\rho_{s s}^{S}=\frac{e^{-\beta E_{s}}}{Z_{S}}
$$

where $Z_{S}=\sum_{s} e^{-\beta E_{s}}$.

After this qualitative discussion, we now show that the precise condition for the Redfield equation to provide an effective description of our master equation is the equivalence between the canonical and microcanonical ensembles. Integrating Eq. (43) over the energy of the environment and using Eq. (37), we get

$$
\begin{aligned}
\dot{\rho}_{S}(t)= & \mathcal{L}_{S} \rho_{S}(t)+\frac{\lambda^{2}}{\hbar^{2}} \sum_{\kappa \kappa^{\prime}} \int_{0}^{t} d \tau \int_{-\infty}^{\infty} d \omega \\
& \times\left\{-e^{-i \omega \tau} \int d \epsilon \widetilde{\alpha}_{\kappa \kappa^{\prime}}(\epsilon, \omega) S^{\kappa} S^{\kappa^{\prime}}(-\tau) P(\epsilon, t)\right. \\
& -e^{i \omega \tau} \int d \epsilon \widetilde{\alpha}_{\kappa^{\prime} \kappa}(\epsilon, \omega) P(\epsilon, t) S^{\kappa^{\prime}}(-\tau) S^{\kappa} \\
& +e^{-i \omega \tau} \int d \epsilon \widetilde{\alpha}_{\kappa^{\prime} \kappa}(\epsilon,-\omega) S^{\kappa} P(\epsilon, t) S^{\kappa^{\prime}}(-\tau)
\end{aligned}
$$

$$
\left.+e^{i \omega \tau} \int d \epsilon \widetilde{\alpha}_{\kappa \kappa^{\prime}}(\epsilon,-\omega) S^{\kappa^{\prime}}(-\tau) P(\epsilon, t) S^{\kappa}\right\} .
$$

In order to close the equation for the subsystem density matrix, we have to assume that the microcanonical correlation functions can be put out of the energy integral. This can only be justified if the environment is such that

$$
\widetilde{\alpha}_{\kappa \kappa^{\prime}}\left(\epsilon+\hbar \omega_{S}, \omega\right) \approx \widetilde{\alpha}_{\kappa \kappa^{\prime}}(\epsilon, \omega) .
$$

By doing this, we obtain Eq. (62), but where the canonical correlation functions have to be replaced by the microcanonical correlation function $\widetilde{\alpha}_{\kappa \kappa^{\prime}}(\beta, \omega) \rightarrow \widetilde{\alpha}_{\kappa \kappa^{\prime}}(\epsilon, \omega)$. Therefore, in order to obtain the Redfield equation, we need to further assume that we can identify the microcanonical with the canonical correlation functions of the environment

$$
\widetilde{\alpha}_{\kappa \kappa^{\prime}}(\epsilon, \omega) \approx \tilde{\alpha}_{\kappa \kappa^{\prime}}(\beta, \omega) .
$$

Let us now find the conditions under which the two assumptions (66) and (67) are valid. The entropy, associated with the microcanonical distribution in an energy shell of the environment of width $\delta_{\epsilon}$ and corresponding to an energy $\epsilon$, is given by

$$
S(\epsilon) \equiv k_{B} \ln w=k_{B} \ln n(\epsilon) \delta_{\epsilon},
$$

where $w$ is the complexion number (i.e., the number of available states in the energy shell). The microcanonical temperature of the environment associated with this microcanonical entropy is defined as

$$
\beta(\epsilon)=\frac{1}{k_{B} T(\epsilon)} \equiv \frac{1}{k_{B}} \frac{d S(\epsilon)}{d \epsilon}=\frac{1}{n(\epsilon)} \frac{d n(\epsilon)}{d \epsilon} .
$$

Suppose that the environment is in a microcanonical distribution at the energy $\epsilon_{m}$. This environment can be effectively described by a canonical distribution at the temperature $\left(k_{B} \beta\right)^{-1}$ if $W(\epsilon)$ is a sharp function with its maximum at the energy $\epsilon_{m}$, which is therefore the most probable energy. In this case, we can expand $\ln W(\epsilon)$ around $\epsilon_{m}$. We get

$$
\begin{aligned}
\ln W(\epsilon)= & \ln W\left(\epsilon_{m}\right)+\left[\frac{d^{2}}{d \epsilon^{2}} \ln W(\epsilon)\right]_{\epsilon=\epsilon_{m}} \frac{\left(\epsilon-\epsilon_{m}\right)^{2}}{2 !} \\
& +\left[\frac{d^{3}}{d \epsilon^{3}} \ln W(\epsilon)\right]_{\epsilon=\epsilon_{m}} \frac{\left(\epsilon-\epsilon_{m}\right)^{3}}{3 !}+\cdots,
\end{aligned}
$$

since $d \ln W\left(\epsilon_{m}\right) / d \epsilon=0$. Because the energy $\epsilon_{m}$ corresponds to the maximum of $\ln W(\epsilon)$, the canonical temperature is equal to the microcanonical temperature at $\epsilon_{m}$

$$
\beta=\beta\left(\epsilon_{m}\right) .
$$

Now using the microcanonical heat capacity defined by

$$
\frac{1}{C(\epsilon)} \equiv \frac{d T(\epsilon)}{d \epsilon}
$$

we have that 


$$
\frac{d^{2}}{d \epsilon^{2}} \ln W(\epsilon)=\frac{d \beta(\epsilon)}{d \epsilon}=-\frac{1}{k_{B} T^{2}(\epsilon) C(\epsilon)} .
$$

We see now that the rational to truncate the series (70) is a large and positive heat capacity. This is true for most environments in the limit of a large number of degrees of freedom $N$, since typically $C(\epsilon) \sim N$. We can now rewrite Eq. (70) as

$$
W(\epsilon) \approx W\left(\epsilon_{m}\right) \exp \left[-\frac{\left(\epsilon-\epsilon_{m}\right)^{2}}{2 \sigma^{2}\left(\epsilon_{m}\right)}\right],
$$

where

$$
\sigma^{2}\left(\epsilon_{m}\right)=k_{B} T^{2}\left(\epsilon_{m}\right) C\left(\epsilon_{m}\right) .
$$

This result confirms that $\epsilon_{m}$ is the maximum of $W(\epsilon)$, and also shows that $\epsilon_{m}$ is its mean value if $\sigma\left(\epsilon_{m}\right)$ is small compared to the typical energies of the environment. This is true for large $N$, since $\epsilon / \sigma\left(\epsilon_{m}\right) \sim \sqrt{N}$. Under these conditions, an environment described by a microcanonical distribution at the energy $\epsilon_{m}$ can be effectively described by a canonical distribution at the temperature $\beta\left(\epsilon_{m}\right)$ so that the second assumption (67) becomes justified. However, in order to justify the first assumption (66), the environment effective canonical temperature has also to remain unchanged under energy shifts of the environment energy of the order of the typical subsystem quanta $\hbar \omega_{S}$

$$
\beta\left(\epsilon \pm \hbar \omega_{S}\right)=\beta(\epsilon) \pm \frac{d \beta(\epsilon)}{d \epsilon} \hbar \omega_{S}+\cdots .
$$

The condition to have such an isothermal environment is

$$
\left|\frac{\hbar \omega_{S}}{\beta(\epsilon)} \frac{d \beta(\epsilon)}{d \epsilon}\right|=\left|\frac{\hbar \omega_{S}}{T(\epsilon) C(\epsilon)}\right| \ll 1 .
$$

This condition is again satisfied when the number of degrees of freedom of the environment becomes large because $\hbar \omega_{S} /[T(\epsilon) C(\epsilon)] \sim 1 / N$. Our conclusion is that, in the limit of an infinitely large environment $N \rightarrow \infty, \sigma(\epsilon)$ becomes infinitely small compared to typical environment energy scales (so that we have the equivalence between the microcanonical and canonical ensembles) but also becomes infinitely large compared to typical subsystem energy scales $\hbar \omega_{S}$ (so that the environment is isothermal for the subsystem). It is in this limit that the Redfield master equation becomes valid and can provide an effective description of our master equation.

\section{CONCLUSIONS}

In this paper, we have considered a subsystem interacting with an environment which has a sufficiently large number of degrees of freedom so that its spectrum can be supposed quasicontinuous. As a consequence, this environment can drive the subsystem into a relaxation process on time scales typically shorter than the Heisenberg time of the environment $\left[t_{H}=\hbar n(\epsilon)\right.$, where $n(\epsilon)$ is the average density of states of the environment]. However, the number of degrees of freedom of this environment may still be too small for the transitions to leave it isothermal. Indeed, the heat capacity is finite and the energy exchanges between the subsystem and the environment can modify the energy distribution of the environment. This is not an unrealistic assumption since the density of states of the environment grows exponentially with the number of degrees of freedom albeit the heat capacity only grows linearly. By using projection operators, we derived a master equation governing the relaxation dynamics of such a subsystem. This equation describes the evolution of the subsystem density matrix distributed over the energy of the environment. This allows us to take into account the changes of the environment energy distribution due to its interaction with the subsystem. By performing the rotating wave approximation (RWA) on this master equation, we have been able to show that the subsystem populations evolve independently from the coherences. The coherences decay in the form of exponentially damped oscillations, while the populations obey a kind of Pauli equation for the total system. This equation conserves the energy of the total system and its equilibrium distribution corresponds to the uniform distribution of the probability over the energy shell of the unperturbed total system. Our equation provides a natural representation of the dynamics of a subsystem interacting with an environment described by a microcanonical distribution. The Redfield master equation is the usual way to represent the dynamics of a subsystem interacting with an environment described by a canonical distribution and is a closed equation for the density matrix of the subsystem. We have shown that, if the equivalence between the microcanonical and canonical ensembles is satisfied for the environment (containing infinitely many degrees of freedom), our equation reduces to the Redfield master equation. If this equivalence is not satisfied, our equation becomes of crucial importance to correctly describe the subsystem relaxation. In the same sense that the microcanonical ensemble is more fundamental than the canonical ensemble, our master equation is more fundamental then the Redfield equations. We believe that this work improves the understanding of kinetic processes in nanosystems where the thermodynamical limit cannot always be taken.

\section{ACKNOWLEDGMENTS}

M.E. thanks the F.R.S.-FNRS Belgium for financial support. This research is financially supported by the "Communauté française de Belgique" (Contract "Actions de Recherche Concertées" No. 04/09-312) and the F.R.S.-FNRS Belgium (Contract F.R.F.C. Nos. 2.4542.02 and 2.4577.04). 
[1] M. Esposito and P. Gaspard, Phys. Rev. E 68, 066112 (2003).

[2] M. Esposito and P. Gaspard, Phys. Rev. E 68, 066113 (2003).

[3] R. Kubo, M. Toda, and N. Saito, Statistical Physics I: Equilibrium Statistical Mechanics, 2nd ed. (Springer, Berlin, 1998).

[4] A. G. Redfield, IBM J. Res. Dev. 1, 19 (1957).

[5] N. G. van Kampen, Stochastic Processes in Physics and Chemistry, 2nd ed. (North-Holland, Amsterdam, 1997).

[6] R. Kubo, M. Toda, and N. Hashitsume, Statistical Physics II: Nonequilibrium Statistical Mechanics, 2nd ed. (Springer, Berlin, 1998).

[7] P. Gaspard and M. Nagaoka, J. Chem. Phys. 111, 5668 (1999).

[8] H.-P. Breuer and F. Petruccione, The Theory of Open Quantum Systems (Oxford University Press, Oxford, 2002).

[9] F. Haake, Statistical Treatment of Open Systems, Springer Tracts in Modern Physics Vol. 66 (Springer, Berlin, 1973).

[10] H. Spohn and J. L. Lebowitz, Adv. Chem. Phys. 38, 109 (1978).

[11] H. Spohn, Rev. Mod. Phys. 53, 569 (1980).

[12] C. Cohen-Tannoudji, J. Dupont-Roc, and G. Grynberg, Processus d'Interaction entre Photons et Atomes (CNRS Editions, Paris, 1996).

[13] C. W. Gardiner and P. Zoller, Quantum Noise (Springer, Berlin, 2000).

[14] R. V. Jensen and R. Shankar, Phys. Rev. Lett. 54, 1879 (1985).

[15] D. H. E. Gross, Microcanonical Thermodynamics: Phase Transitions in "Small" Systems (World Scientific, Singapore, 2000).

[16] J. Jellinek and A. Goldberg, J. Chem. Phys. 113, 2570 (2000).

[17] R. B. Shirts and M. R. Shirts, J. Chem. Phys. 117, 5564 (2002).

[18] D. H. E. Gross and J. F. Kenney, J. Chem. Phys. 122, 224111
(2005)

[19] C. Junghans, M. Bachmann, and W. Janke, Phys. Rev. Lett. 97, 218103 (2006).

[20] J. Barré, D. Mukamel, and S. Ruffo, Phys. Rev. Lett. 87, 030601 (2001).

[21] Nonextensive systems; see I. Ispolatov and E. G. D. Cohen, Physica A 295, 475 (2001).

[22] G. Benenti, G. Casati, and D. L. Shepelyansky, Eur. Phys. J. D 17, 265 (2001).

[23] J. Barré, Physica A 305, 172 (2002).

[24] P. Hertel and W. Thirring, Ann. Phys. (N.Y.) 63, 520 (1971).

[25] D. H. E. Gross, Phys. Rep. 279, 119 (1997).

[26] M. Antoni, S. Ruffo, and A. Torcini, Phys. Rev. E 66, 025103(R) (2002).

[27] H. Touchette, R. S. Ellis, and B. Turkington, Physica A 340, 138 (2004).

[28] S. Mukamel, Principles of Nonlinear Optical Spectroscopy (Oxford University Press, New York, 1995).

[29] G. Lindblad, Commun. Math. Phys. 48, 119 (1976).

[30] M. Esposito, e-print arXiv:cond-mat/0412495.

[31] A. A. Budini, Phys. Rev. A 74, 053815 (2006).

[32] H. P. Breuer, J. Gemmer, and M. Michel, Phys. Rev. E 73, 016139 (2006).

[33] H. P. Breuer, Phys. Rev. A 75, 022103 (2007).

[34] A. Suarez, R. Silbey, and I. Oppenheim, J. Chem. Phys. 97, 5101 (1992).

[35] Y. C. Cheng and R. J. Silbey, J. Phys. Chem. B 109, 21399 (2005).

[36] D. Kohen, C. C. Marston, and D. J. Tannor, J. Chem. Phys. 107, 5236 (1997). 